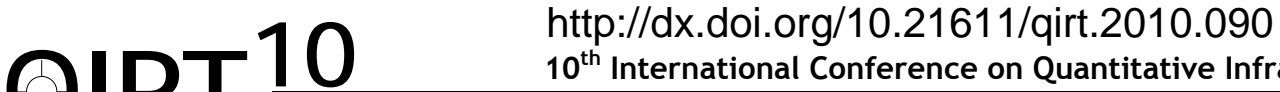 \\ $10^{\text {th }}$ International Conference on Quantitative InfraRed Thermography \\ July 27-30, 2010, Québec (Canada)
}

\section{Temperature measurement at large distance of plasma-facing surfaces in fusion reactor by active pyrometry}

\author{
by D. Melyukov*, C. Sortais*, D. Farcage*, A. Semerok*, P.-Y. Thro* and E. Gauthier** \\ ${ }^{*}$ CEA Saclay, DEN/DANS/DPC/SCP/LILM, Gif-surYvette, France, dmitry.melyukov@cea.fr \\ ${ }^{*}$ CEA Cadarache, DSM/IRFM/SIPP, Saint Paul lez Durance, France, eric.gauthier@cea.fr
}

\begin{abstract}
The aim of the present work is to demonstrate in laboratory conditions that we can measure the surface temperature of metallic substrates. For highly reflecting environments, the bicolour pyrometry seems to be the appropriate technique. It consists of temperature measurements deduced by the ratio between two modulated signals.

We built an experimental set-up at long distance ( $2.15 \mathrm{~m}$ between the sample and the detector) to be as similar as possible as tokamak conditions. We proved experimentally the feasibility, at long distance and with some artificial reflective fluxes, of our technique working on graphite, ITER-relevant samples and stainless steel.
\end{abstract}

\section{Introduction}

The aim of this work is to measure the surface temperature of a metallic sample at a long distance by means of pulsed laser photo-thermal method. It is considered as a possible technique to the future ITER reactor.

Presence of plasma inside tokamak-type reactor defines special requirements on wall materials. Presently, most of Plasma Facing Materials are made of carbon. In the future, carbon should be replaced by Tungsten and Beryllium in order to minimize the Tritium retention. Therefore JET will be operated next year with Carbon Fiber Composite coated with $10 \mu \mathrm{m}$ tungsten layer. In normal working conditions, their temperature varies from $300^{\circ} \mathrm{C}$ to $1500^{\circ} \mathrm{C}$ and, in any case, must remain much below the fusion temperature of the wall. To provide efficient working conditions for tokamaks, an accurate measurement of temperature is then needed. As this technique is aimed for ITER, we want it to be contactless, nondestructive and remote. We also want it to be independent of emissivity as its exact value may vary widely as a function of roughness, contamination, oxidation... Moreover, operating in a highly reflecting environment, this technique must be independent of the reflected flux.

All these requirements can be satisfied by using a surface temperature measurement theoretically and experimentally developed by T. Loarer [1-3]. Unlike the classical pyrometry operating with a single detector, the proposed method registers the temperature by two detectors working at different mean wavelengths. The studied surface is subjected to a small thermal perturbation aimed to modulate its temperature. As a result each of the optical detectors records modulated signal. The temperature deduced by the ratio between the two modulated signals is independent on the environmental reflected fluxes as well as on the surface emissivity.

Previous studies [2-5] were done at short distance between detectors and samples. The aim of the present study was the realization and the test of an experimental laboratory setup of the method at long distance (typically $2 \mathrm{~m}$ ), which further to be used for in-situ measurements at JET.

In section 2, we present the theoretical background of our study. The experimental setup is described in section 3. In order to interpret the thermal signals correctly, a calibration of our pyrometer is needed (section 4). We finally discuss of our experimental results in section 5 before conclusions and discussions in section 6 .

\section{Theoretical background}

The signal measured by an infra-red detector is the sum of two components: the flux emitted by the heated sample $\Phi \mathrm{e}$ and the reflecting flux due to the environment $\Phi \mathrm{r}$. The emitted flux can be written:

$$
\Phi e=\varepsilon(\lambda, T) \times L(\lambda, T)
$$

where $\varepsilon(\lambda, T)$ is the emissivity and $L(\lambda, T)\left(\mathrm{Wm}^{-3} \mathrm{str}^{-1}\right)$ is the black-body spectral radiance at certain wavelength $\lambda(\mathrm{m})$ and certain temperature $\mathrm{T}(\mathrm{K})$. It follows the Planck's law: 


$$
\Phi e=\int_{\lambda_{i}-\frac{\Delta \lambda_{i}}{2}}^{\lambda_{i}+\frac{\Delta \lambda_{i}}{2}} \varepsilon(\lambda, T) \times \frac{C_{1}}{\lambda_{i}^{5} \times\left[\exp \left(\frac{C_{2}}{\lambda_{i} \times T}\right)-1\right]} d \lambda \quad \text { with }\left\{\begin{array}{l}
C_{1}=2 \times h \times c^{2}=1.19106 \times 10^{-16} W . m \\
C_{2}=\frac{h \times c}{k_{B}}=1.4388 \times 10^{-2} m K \\
\lambda_{i}(m) \text { mean wavelength and } \Delta \lambda_{i} \text { filter bandwidth }
\end{array}\right.
$$

To proceed, we assume that the surface is grey (i.e. $\varepsilon(\lambda)=$ constant at least over the filter's bandwidth range). A discussion of the error introduced by this assumption can be found in [1]. We also assume that, the bandwidth of our interferential filters being small enough, we verify the relation:

$$
\int L\left(\lambda_{i}, T\right) d \lambda \approx \Delta \lambda_{i} \times L\left(\lambda_{i}, T\right)
$$

with $\lambda_{\mathrm{i}}$ : central wavelength of interferential filter.

The total flux $\Phi$ leaving the surface at temperature $T_{0}$ is then:

$$
\Phi=\varepsilon\left(T_{0}\right) \times \Delta \lambda_{i} \times \frac{C_{1}}{\lambda_{i}^{5} \times\left[\exp \left(\frac{C_{2}}{\lambda_{i} \times T_{0}}\right)-1\right]}+\Phi r\left(T_{0}\right)
$$

If one modulates the temperature by locally illuminating the surface with a pulsed laser beam, the surface temperature becomes: $T=T_{0}+\Delta T$. If $\Delta T$ is small $(\Delta T<10 \mathrm{~K})$, then the reflected fluxes and emissivity can be assumed constant.

Hence:

$$
\Delta \Phi_{i}=\varepsilon\left(T_{0}\right) \times \Delta \lambda_{i} \times \int_{T_{0}}^{T_{0}+\Delta T} \frac{\partial L}{\partial T} d T
$$

We consider the approximation of the Planck law at first order then the above equation becomes:

$$
\Delta \Phi_{i}=\varepsilon\left(T_{0}\right) \times \Delta \lambda_{i} \times \Delta T \times \frac{\partial L}{\partial T}
$$

During our experiments, we record a signal modulation $\triangle \mathrm{R}$ proportional to $\Delta \Phi$ as follows:

$$
\Delta R_{i}=A \times \Omega \times r \times \varepsilon_{\lambda} \times D_{\lambda} \times \tau_{\lambda} \times \Delta \Phi_{i}
$$

where: A, $m^{2}$ - perturbed zone surface; $\Omega$,str - pyrometer's solid angle; r,VA ${ }^{-1}$ - pyrometer's electrical resistance; $D_{\lambda}$, $A W^{-1}$ - detector's sensitivity; $T_{\lambda}$ - optical transmissivity at wavelength $\lambda ; \Delta \lambda, \mathrm{m}$ - pyrometer's spectral bandwidth.

The thermal evolution of the heated body will be followed at two different wavelength $\lambda_{1}$ and $\lambda_{2}$. Mathematically, the ratio is independent of reflected fluxes and, assuming that we deal with a grey body, independent of emissivity:

$$
\frac{\Delta R_{2}}{\Delta R_{1}}=\frac{D_{\lambda_{2}} \times \tau_{\lambda_{2}} \times \Delta \lambda_{2} \times \frac{\partial}{\partial T} L\left(\lambda_{2}, T_{0}\right)}{D_{\lambda_{1}} \times \tau_{\lambda_{1}} \times \Delta \lambda_{1} \times \frac{\partial}{\partial T} L\left(\lambda_{1}, T_{0}\right)}
$$

\section{Experimental set-up}

The experimental set-up consists of a pyrometer Kleiber 740-LO, two external interferential bandpass filters, an infra-red Nd:YAG laser and a sample (either graphite, ITER-relevant sample or 304L stainless steel) (figure 1). The pyrometer operates in the range $350^{\circ} \mathrm{C}-1000^{\circ} \mathrm{C}$ and in the spectral range $1.58 \mu \mathrm{m}-2.20 \mu \mathrm{m}$, with a response time of $10 \mu \mathrm{s}$. The two external band pass filters have the following characteristics:

-filter 1 has a mean wavelength of $1669 \mathrm{~nm}$, a mean spectral bandwidth of $29 \mathrm{~nm}$ and a maximal transmissivity of $84 \%$, -filter 2 has a mean wavelength of $2085 \mathrm{~nm}$, a mean spectral bandwidth of $83 \mathrm{~nm}$ and a maximal transmissivity of $83 \%$. 
The pulsed Nd:YAG laser is a Q-switched Quantel Brilliant. It has a repetition rate of $20 \mathrm{~Hz}$, a pulse duration of $120 \mu \mathrm{s}$ and a laser-pulse energy varying from 10.5 to $400 \mathrm{~mJ}$.



Fig. 1. Scheme of the experimental set-up

Three types of sample were used during our experiments: (i) a graphite circle of $4 \mathrm{~cm}$-diameter and $2 \mathrm{~mm}$-tickness, (ii) an ITER-relevant sample made of a stainless steel substrate with a $3 \mu \mathrm{m}$-thick carbon and tungsten layer, (iii) a $304 \mathrm{~L}$ stainless steel block $\left(25 \mathrm{~cm}^{2}, 1 \mathrm{~cm}\right.$-thick). The studied sample has to be heated so that the temperature is high enough to be recorded by the pyrometer $\left(T>350^{\circ} \mathrm{C}\right)$. A second reason for heating the sample is to be representative of the temperature conditions on the tokamak wall. In this work, we realized heating thanks to a Joule-effect heater. The device which enables heating is also the sample holder. All the samples were positioned $2.15 \mathrm{~m}$ far from the pyrometer and the laser.

\section{Pyrometer calibration}

Pyrometer calibrations were performed under ambient conditions using a black-body temperature range between $300^{\circ} \mathrm{C}$ and $900^{\circ} \mathrm{C}$. Obtained signals were recorded with an oscilloscope. By processing the experimental data, we established the $R=f(T)$ functions of the calibration equations, using a Planck type function:

$$
R_{i}=Z_{i} \times \frac{X_{i}}{\left[\exp \left(\frac{Y_{i}}{T}\right)-1\right]}
$$

The calibration constant values for each system (pyrometer + filter i) are listed in table 1.

Table 1. Calibration parameters obtained after fit convergence

\begin{tabular}{|l|l|}
\hline \multicolumn{1}{|c|}{ Pyrometer + filter 1 } & \multicolumn{1}{|c|}{ Pyrometer + filter 2 } \\
\hline$Z_{1}=(3.057 \pm 0.0009) \times 10^{-10} \mathrm{~V} \cdot \mathrm{J}^{-1} \cdot \mathrm{m}^{3} \cdot \mathrm{s}$ & $\mathrm{Z}_{2}=(8.450 \pm 0.0008) \times 10^{-10} \mathrm{~V} \cdot \mathrm{J}^{-1} \cdot \mathrm{m}^{3} \cdot \mathrm{s}$ \\
\hline $\mathrm{X}_{1}=9.197 \times 10^{12} \mathrm{~J} \cdot \mathrm{m}^{3} \cdot \mathrm{s}^{-1}$ & $\mathrm{X}_{2}=3.023 \times 10^{12} \mathrm{~J} \cdot \mathrm{m}^{3} \cdot \mathrm{s}^{-1}$ \\
\hline $\mathrm{Y}_{1}=8621 \mathrm{~K}$ & $\mathrm{Y}_{2}=6901 \mathrm{~K}$ \\
\hline
\end{tabular}

Eq. (6) is visualised in figure 2 and figure 3 for the (pyrometer + filter 1 ) system and the (pyrometer + filter 2 ) system, respectively. 


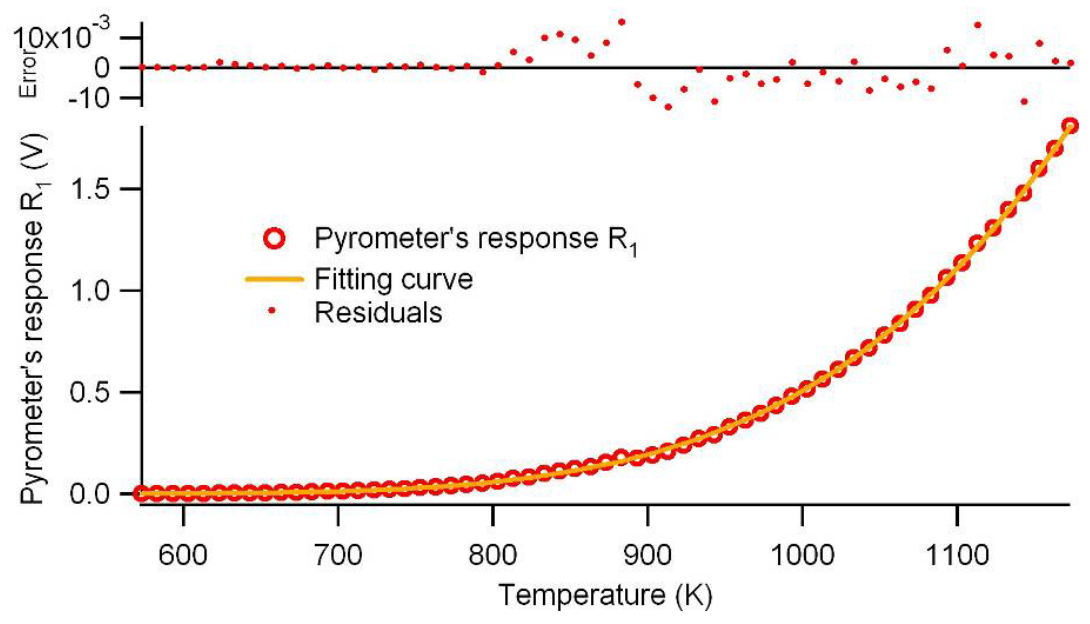

Fig. 2. Calibration curve $R_{1}=f(T)$ for the (pyrometer + filter 1$)$ system

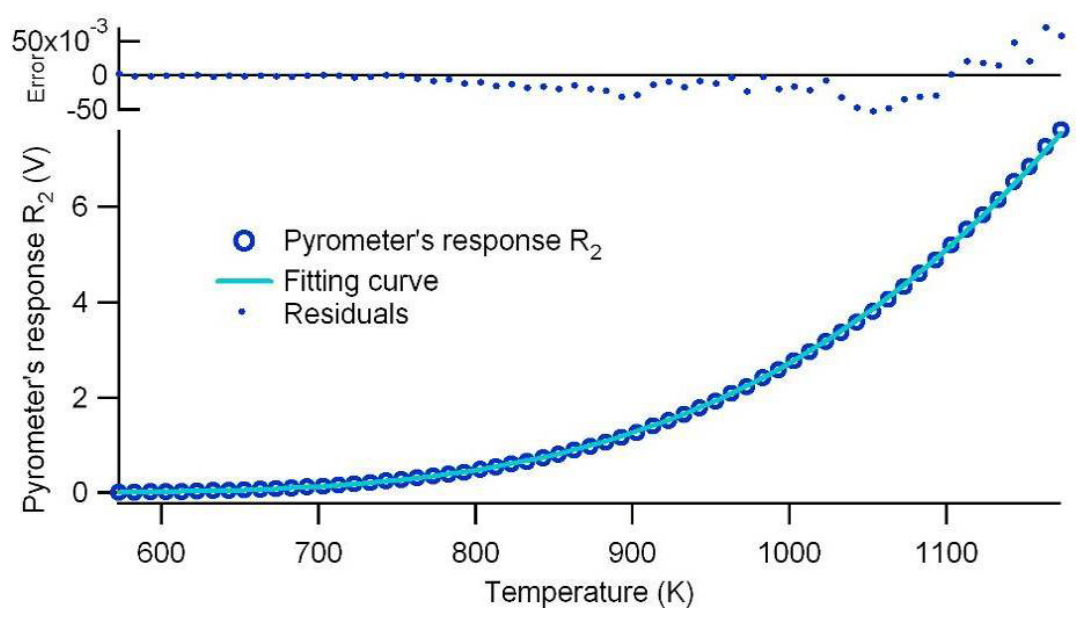

Fig. 3. Calibration curve $R_{2}=f(T)$ for the (pyrometer + filter 2) system

The calibration procedure gives us empirical numerical relation between signal and temperature for both filters. Mathematically speaking and saying that $\Delta \mathrm{T} \rightarrow 0$, the relation between signal modulation and temperature is the derivative in respect to temperature of the calibration equations in their $R_{i}=f(T)$ mode:

$$
\Delta R_{i}=\Delta T \times \frac{\partial R_{i}}{\partial T}
$$

Hence the ratio curve may be computed as follows:

$$
\frac{\Delta R_{2}}{\Delta R_{1}}=\frac{\partial R_{2}}{\partial T} / \frac{\partial R_{1}}{\partial T}
$$

The final expression of the calibration curve is: 


$$
\frac{\Delta R_{2}}{\Delta R_{1}}=\frac{Z_{2} \times X_{2} \times Y_{2}}{Z_{1} \times X_{1} \times Y_{1}} \times \exp \left(\frac{Y_{2}-Y_{1}}{T}\right) \times\left[\frac{\exp \left(\frac{Y_{1}}{T}\right)-1}{\exp \left(\frac{Y_{2}}{T}\right)-1}\right]^{2}
$$

The calibration curve is represented on figure 4 .

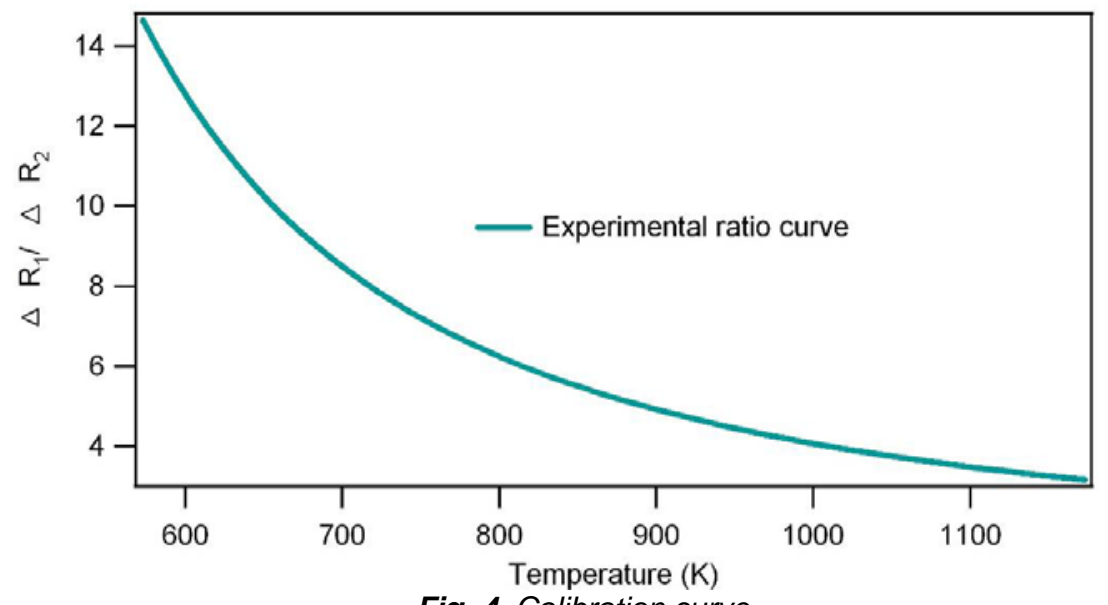

Fig. 4. Calibration curve

\section{Results and analyses}

Here we wanted to be as close as possible to the conditions found inside a tokamak. Experimental data were then collected on the three samples at $2.15 \mathrm{~m}$ far from the pyrometer. Experiments were done without and/or with additional parasite flux, to simulate the highly reflective fluxes in tokamaks.

\subsection{Experiments without reflective flux and at large distance}

The results obtained on the three samples described above are represented on figure 5, figure 6 and figure 7. Each experiment was performed at large distance. The laser power needs to be suited to the sample type to induce a thermal perturbation that can be measured by our system.

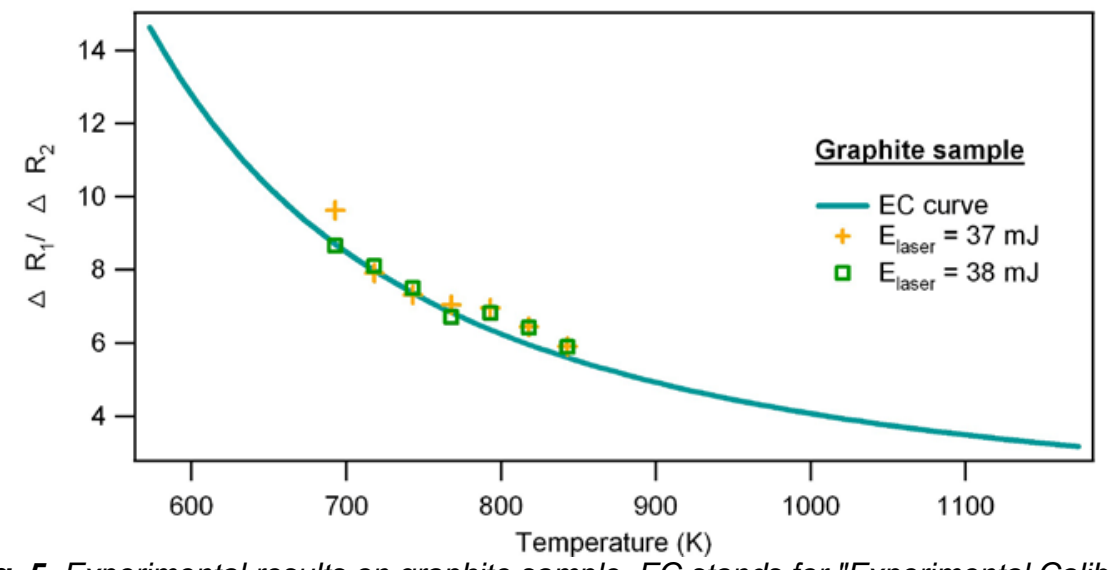

Fig. 5. Experimental results on graphite sample. EC stands for "Experimental Calibration". 


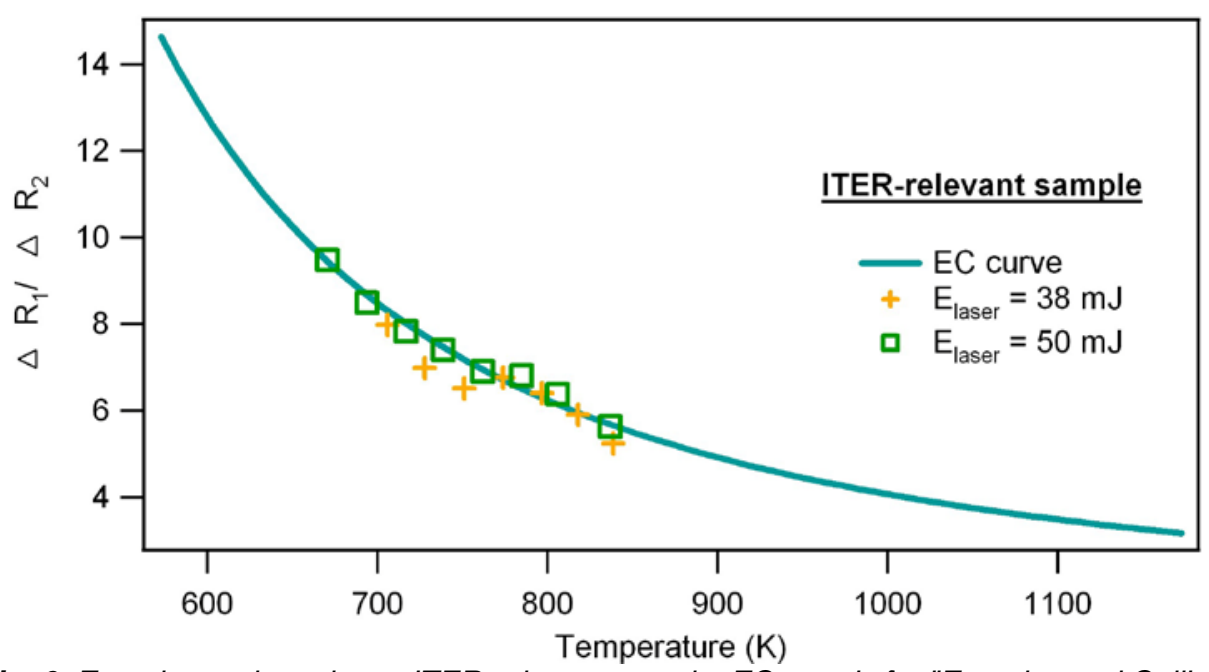

Fig. 6. Experimental results on ITER-relevant sample. EC stands for "Experimental Calibration".

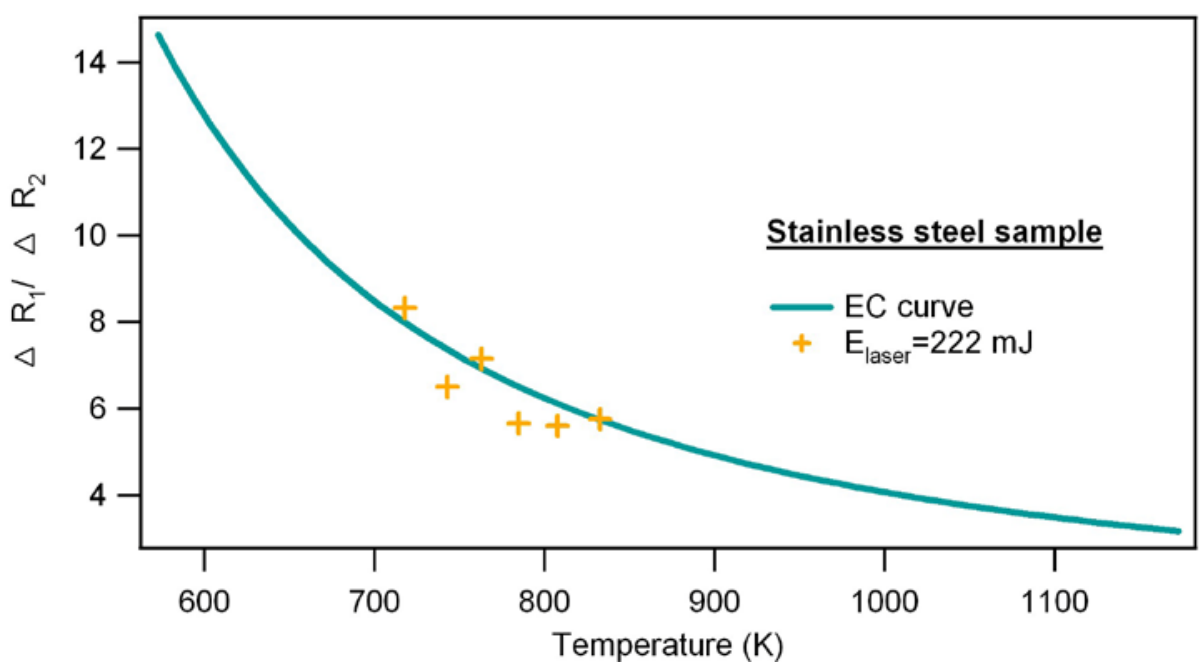

Fig. 7. Experimental results on stainless steel sample. EC stands for "Experimental Calibration".

In the case of graphite and ITER-relevant samples, the bicolour pyrometry gives good results at large distance. As for the stainless steel sample, the technique gives good qualitative results but quantitatively has still to be improved.

\subsection{Experiments with reflective flux}

The reflective flux was obtained thanks to a blackbody cavity heated up to $900^{\circ} \mathrm{C}$. The typical signal measured with the reflective flux is 2 times the signal measured without reflective flux, i.e. the situation is representative of the inside conditions in a tokamak. The data were measured with an oscilloscope (table 2). The electrical signals measured for the (pyrometer+filter 1 ) system are very low (only several $\mathrm{mV}$ ). As a consequence, the uncertainties are big and the calculated ratio can vary widely, preventing us from concluding on this point of our study.

At this stage of our work we have to change our detector. This part of our work will be hopefully developed during our oral presentation. 
Table 2. Experimental data obtained on stainless steel sample with a reflective flux

\begin{tabular}{|c|c|c|c|c|c|}
\hline filter & $\Delta R(\mathrm{mV})$ & Ratio & $\mathrm{T}\left({ }^{\circ} \mathrm{C}\right)$ - measured & $\mathrm{T}\left({ }^{\circ} \mathrm{C}\right)$-theory & $\mathrm{T}\left({ }^{\circ} \mathrm{C}\right)$ - blackbody \\
\hline 1 & 4 & \multirow{2}{*}{6.50} & \multirow{2}{*}{520} & \multirow{2}{*}{420} & \multirow{2}{*}{600} \\
\hline 2 & 26 & & & & \\
\hline 2 & 21.3 & \multirow{2}{*}{6.17} & \multirow{2}{*}{545} & \multirow{2}{*}{420} & \multirow{2}{*}{0} \\
\hline 1 & 3.45 & & & & \\
\hline 1 & 3.2 & \multirow{2}{*}{8.13} & \multirow{2}{*}{440} & \multirow{2}{*}{420} & \multirow{2}{*}{900} \\
\hline 2 & 26 & & & & \\
\hline 2 & 19.6 & \multirow{2}{*}{7.84} & \multirow{2}{*}{455} & \multirow{2}{*}{375} & \multirow{2}{*}{300} \\
\hline 1 & 2.5 & & & & \\
\hline 1 & 2.43 & \multirow{2}{*}{7.33} & \multirow{2}{*}{475} & \multirow{2}{*}{375} & \multirow{2}{*}{0} \\
\hline 2 & 17.8 & & & & \\
\hline 2 & 16 & \multirow{2}{*}{5.71} & \multirow{2}{*}{575} & \multirow{2}{*}{375} & \multirow{2}{*}{800} \\
\hline 1 & 2.8 & & & & \\
\hline 1 & 5.85 & \multirow{2}{*}{6.50} & \multirow{2}{*}{520} & \multirow{2}{*}{465} & \multirow{2}{*}{440} \\
\hline 2 & 38 & & & & \\
\hline 2 & 35.6 & \multirow{2}{*}{5.86} & \multirow{2}{*}{560} & \multirow{2}{*}{465} & \multirow{2}{*}{0} \\
\hline 1 & 6.08 & & & & \\
\hline 1 & 5.1 & \multirow{2}{*}{7.84} & \multirow{2}{*}{455} & \multirow{2}{*}{465} & \multirow{2}{*}{880} \\
\hline 2 & 40 & & & & \\
\hline
\end{tabular}

\section{Conclusions}

Aiming to validate our laboratory set-up for temperature measurement, we tested two different parameters of the method: (i) the distance between the sample and the detector, (ii) the presence of reflective fluxes.

We proved that the bicolour pyrometry is a good technique to determine surface temperature at large distance. Concerning the experiments with reflected flux, we obtained encouraging results on the stainless steel sample, which have to be realised by using a more suitable pyrometer.

\section{REFERENCES}

[1] Loarer T., "Mesure de température de surface par effet photothermique module ou impulsionnel," Ecole Centrale (France), 1989.

[2] Loarer T., Greffet J.-J., Applied Optics, 31, p. 5350-5358, 1992.

[3] Loarer T., Greffet J.-J., Huetz-Aubert M., Applied Optics, 29, p. 979-987, 1990.

[4] Grigorova V. et al., "Active IR thermography by means of pulsed photothermal effect", EFDA report JW6-FT3.37, 62 p., 2007.

[5] Grigorova V. et al., Journal of nuclear materials, 390-391, p. 1097-1101, 2009. 